\title{
An Inverse Eigenvalue Problem of Hermite-Hamilton Matrices in Structural Dynamic Model Updating
}

\author{
Linlin Zhao and Guoliang Chen \\ Department of Mathematics, East China Normal University, Shanghai 200241, China \\ Correspondence should be addressed to Guoliang Chen, glchen@math.ecnu.edu.cn
}

Received 11 February 2010; Accepted 27 April 2010

Academic Editor: Angelo Luongo

Copyright (c) 2010 L. Zhao and G. Chen. This is an open access article distributed under the Creative Commons Attribution License, which permits unrestricted use, distribution, and reproduction in any medium, provided the original work is properly cited.

We first consider the following inverse eigenvalue problem: given $X \in C^{n \times m}$ and a diagonal matrix $\Lambda \in C^{m \times m}$, find $n \times n$ Hermite-Hamilton matrices $K$ and $M$ such that $K X=M X \Lambda$. We then consider an optimal approximation problem: given $n \times n$ Hermitian matrices $K_{a}$ and $M_{a}$, find a solution $(K, M)$ of the above inverse problem such that $\left\|K-K_{a}\right\|^{2}+\left\|M-M_{a}\right\|^{2}=\min$. By using the MoorePenrose generalized inverse and the singular value decompositions, the solvability conditions and the representations of the general solution for the first problem are derived. The expression of the solution to the second problem is presented.

\section{Introduction}

Throughout this paper, we will adopt the following notations. Let $C^{m \times n}, H C^{n \times n}$, and $U C^{n \times n}$ stand for the set of all $m \times n$ matrices, $n \times n$ Hermitian matrices, and unitary matrices over the complex field $C$, respectively. By $\|\cdot\|$ we denote the Frobenius norm of a matrix. The symbols $A^{T}, A^{*}, A^{-1}$, and $A^{\dagger}$ denote the transpose, conjugate transpose, inverse, and Moore-Penrose generalized inverse of $A$, respectively.

Definition 1.1. Let $J_{n}=\left(\begin{array}{cc}0 & I_{k} \\ -I_{k} & 0\end{array}\right), n=2 k$, and $A \in C^{n \times n}$. If $A=A^{*}$ and $J_{n} A J_{n}=A^{*}$, then the matrix $A$ is called Hermite-Hamilton matrix.

We denote by $H H^{n \times n}$ the set of all $n \times n$ Hermite-Hamilton matrices.

Vibrating structures such as bridges, highways, buildings, and automobiles are modeled using finite element techniques. These techniques generate structured matrix second-order differential equations:

$$
M_{a} \ddot{z}(t)=K_{a} z(t)
$$


where $M_{a}, K_{a}$ are analytical mass and stiffness matrices. It is well known that all solutions of the above differential equation can be obtained via the algebraic equation $K_{a} x=\lambda M_{a} x$. But such finite element model is rarely available in practice, because its natural frequencies and mode shapes often do not match very well with experimentally measured ones obtained from a real-life vibration test [1]. It becomes necessary to update the original model to attain consistency with empirical results. The most common approach is to modify $K_{a}$ and $M_{a}$ to satisfy the dynamic equation with the measured model data. Let $X \in C^{n \times m}$ be the measured model matrix and $\Lambda=\operatorname{diag}\left(\delta_{1}, \delta_{2}, \ldots, \delta_{m}\right) \in C^{m \times m}$ the measured natural frequencies matrix, where $n \geq m$. The measured mode shapes and frequencies are assumed correct and have to satisfy

$$
K X=M X \Lambda,
$$

where $M, K \in C^{n \times n}$ are the mass and stiffness matrices to be corrected. To date, many techniques for model updating have been proposed. For undamped systems, various techniques have been discussed by Berman [2] and Wei [3]. Theory and computation of damped systems were proposed by authors of [4,5]. Another line of thought is to update damping and stiffness matrices with symmetric low-rank correction [6]. The system matrices are adjusted globally in these methods. As model errors can be localized by using sensitivity analysis [7], residual force approach [8], least squares approach [9], and assigned eigenstructure [10], it is usual practice to adjust partial elements of the system matrices using measured response data.

The model updating problem can be regarded as a special case of the inverse eigenvalue problem which occurs in the design and modification of mass-spring systems and dynamic structures. The symmetric inverse eigenvalue problem and generalized inverse eigenvalue problem with submatrix constraint in structural dynamic model updating have been studied in [11] and [12], respectively. Hamiltonian matrices usually arise in the analysis of dynamic structures [13]. However, the inverse eigenvalue problem for Hermite-Hamilton matrices has not been discussed. In this paper, we will consider the following inverse eigenvalue problem and an associated optimal approximation problem.

Problem 1. Given that $X \in C^{n \times m}$ and a diagonal matrix $\Lambda \in C^{m \times m}$, find $n \times n$ Hermite-Hamilton matrices $K$ and $M$ such that

$$
K X=M X \Lambda .
$$

Problem 2. Given that $K_{a}, M_{a} \in H C^{n \times n}$, let $S_{E}$ be the solution set of Problem 1. Find $(\widehat{K}, \widehat{M}) \in$ $S_{E}$ such that

$$
\left\|\widehat{K}-K_{a}\right\|^{2}+\left\|\widehat{M}-M_{a}\right\|^{2}=\min _{(K, M) \in S_{E}}\left(\left\|K-K_{a}\right\|^{2}+\left\|M-M_{a}\right\|^{2}\right) .
$$

We observe that, when $M=I$, Problem 1 can be reduced to the following inverse eigenproblem:

$$
K X=X \Lambda,
$$


which has been solved for different classes of structured matrices. For example, Xie et al. considered the problem for the case of symmetric, antipersymmetric, antisymmetric, and persymmetric matrices in [14, 15]. Bai and Chan studied the problem for the case of centrosymmetric and centroskew matrices in [16]. Trench investigated the case of generalized symmetry or skew symmetry matrices for the problem in [17] and Yuan studied R-symmetric matrices for the problem in [18].

The paper is organized as follows. In Section 2, using the Moore-Penrose generalized inverse and the singular value decompositions of matrices, we give explicit expressions of the solution for Problem 1. In Section 3, the expressions of the unique solution for Problem 2 are given and a numerical example is provided.

\section{Solution of Problem 1}

Let

$$
U=\frac{1}{\sqrt{2}}\left(\begin{array}{cc}
I_{k} & I_{k} \\
-i I_{k} & i I_{k}
\end{array}\right) .
$$

Lemma 2.1. Let $A \in C^{n \times n}$. Then $A \in H H C^{n \times n}$ if and only if there exists a matrix $N \in C^{k \times k}$ such that

$$
A=U\left(\begin{array}{cc}
0 & N \\
N^{*} & 0
\end{array}\right) U^{*},
$$

where $U$ is the same as in (2.1).

Proof. Let $A=\left(\begin{array}{ll}A_{11} & A_{12} \\ A_{12}^{*} & A_{22}\end{array}\right)$, and let each block of $A$ be square. From Definition 1.1 and (2.1), it can be easily proved.

Lemma 2.2 (see [19]). Let $A \in C^{m \times n}, B \in C^{p \times q}$, and $E \in C^{m \times q}$. Then the matrix equation $A X B=E$ has a solution $X \in C^{n \times p}$ if and only if $A A^{\dagger} E B^{\dagger} B=E$; in this case the general solution of the equation can be expressed as $X=A^{\dagger} E B^{\dagger}+Y-A^{\dagger} A Y B B^{\dagger}$, where $Y \in C^{n \times p}$ is arbitrary.

Let the partition of the matrix $U^{*} X$ be

$$
U^{*} X=\left(\begin{array}{l}
X_{1} \\
X_{2}
\end{array}\right), \quad X_{1}, X_{2} \in C^{k \times m},
$$

where $U$ is defined as in (2.1).

We assume that the singular value decompositions of the matrices $X_{1}$ and $X_{2}$ are

$$
X_{1}=R\left(\begin{array}{ll}
D & 0 \\
0 & 0
\end{array}\right) S^{*}, \quad X_{2}=W\left(\begin{array}{ll}
\Sigma & 0 \\
0 & 0
\end{array}\right) V^{*},
$$


where $R=\left(R_{1}, R_{2}\right) \in U C^{k \times k}, S=\left(S_{1}, S_{2}\right) \in U C^{m \times m}, D=\operatorname{diag}\left(d_{1}, \ldots, d_{l}\right)>0, l=$ $\operatorname{rank}\left(X_{1}\right), R_{1} \in C^{k \times l}, S_{1} \in C^{m \times l}$, and $W=\left(W_{1}, W_{2}\right) \in U C^{k \times k}, V=\left(V_{1}, V_{2}\right) \in U C^{m \times m}$, $\Sigma=\operatorname{diag}\left(\sigma_{1}, \ldots, \sigma_{s}\right)>0, s=\operatorname{rank}\left(X_{2}\right), W_{1} \in C^{k \times s}, V_{1} \in C^{m \times s}$.

Let the singular value decompositions of the matrices $X_{2} \Lambda V_{2}$ and $X_{1} \Lambda S_{2}$ be

$$
X_{2} \Lambda V_{2}=P\left(\begin{array}{ll}
\Omega & 0 \\
0 & 0
\end{array}\right) Q^{*}, \quad X_{1} \Lambda S_{2}=T\left(\begin{array}{ll}
\Delta & 0 \\
0 & 0
\end{array}\right) H^{*}
$$

where $P=\left(P_{1}, P_{2}\right) \in U C^{k \times k}, Q=\left(Q_{1}, Q_{2}\right) \in U C^{(m-s) \times(m-s)}, \Omega=\operatorname{diag}\left(\omega_{1}, \ldots, \omega_{t}\right)>0, t=$ $\operatorname{rank}\left(X_{2} \Lambda V_{2}\right), P_{1} \in C^{k \times t}, Q_{1} \in C^{(m-s) \times t}$, and $T=\left(T_{1}, T_{2}\right) \in U C^{k \times k}, H \in U C^{(m-l) \times(m-l)}, \Delta=$ $\operatorname{diag}\left(a_{1}, \ldots, a_{g}\right)>0, g=\operatorname{rank}\left(X_{1} \Lambda S_{2}\right), T_{1} \in C^{k \times g}$.

Theorem 2.3. Suppose that $X \in C^{n \times m}$ and $\Lambda \in C^{m \times m}$ is a diagonal matrix. Let the partition of $U^{*} X$ be (2.3), and let the singular value decompositions of $X_{1}, X_{2}, X_{2} \Lambda V_{2}$, and $X_{1} \Lambda S_{2}$ be given in (2.4) and (2.5), respectively. Then (1.3) is solvable and its general solution can be expressed as

$$
M=U\left(\begin{array}{cc}
0 & F \\
F^{*} & 0
\end{array}\right) U^{*}, \quad K=U\left(\begin{array}{cc}
0 & F X_{2} \Lambda X_{2}^{\dagger}+G W_{2}^{*} \\
\left(F X_{2} \Lambda X_{2}^{\dagger}+G W_{2}^{*}\right)^{*} & 0
\end{array}\right) U^{*}
$$

where

$$
F=T_{2} J P_{2}^{*}, \quad G=\left(X_{1} \Lambda X_{1}^{\dagger}\right)^{*} F W_{2}+R_{2} Y
$$

with $J \in C^{(k-g) \times(k-t)}, Y \in C^{(k-l) \times(k-s)}$ being arbitrary matrices, and $U$ is the same as in (2.1).

Proof. By Lemma 2.1, we know that $(K, M)$ is a solution to Problem 1 if and only if there exist matrices $N, F \in C^{k \times k}$ such that

$$
\begin{gathered}
K=U\left(\begin{array}{cc}
0 & N \\
N^{*} & 0
\end{array}\right) U^{*}, \quad M=U\left(\begin{array}{cc}
0 & F \\
F^{*} & 0
\end{array}\right) U^{*}, \\
U\left(\begin{array}{cc}
0 & N \\
N^{*} & 0
\end{array}\right) U^{*} X=U\left(\begin{array}{cc}
0 & F \\
F^{*} & 0
\end{array}\right) U^{*} X \Lambda .
\end{gathered}
$$

Using (2.3), the above equation is equivalent to the following two equations:

$$
\begin{gathered}
N X_{2}=F X_{2} \Lambda, \\
N^{*} X_{1}=F^{*} X_{1} \Lambda, \quad \text { i.e., } X_{1}^{*} N=\left(X_{1} \Lambda\right)^{*} F .
\end{gathered}
$$

By the singular value decomposition of $X_{2}$, then the relation (2.9) becomes

$$
\begin{gathered}
0=F X_{2} \Lambda V_{2}, \\
N W_{1} \Sigma=F X_{2} \Lambda V_{1} .
\end{gathered}
$$


Clearly, (2.11) with respect to unknown matrix $F$ is always solvable. By Lemma 2.2 and (2.5), we get

$$
F=L P_{2}^{*}
$$

where $L \in C^{k \times(k-t)}$ is an arbitrary matrix. Substituting $F=L P_{2}^{*}$ into (2.12), we get

$$
N W_{1}=\left(L P_{2}^{*}\right) X_{2} \Lambda V_{1} \Sigma^{-1}
$$

Since $W_{1}$ is of full column rank, then the above equation with respect to unknown matrix $N$ is always solvable, and the general solution can be expressed as

$$
\begin{aligned}
N & =\left(L P_{2}^{*} X_{2} \Lambda V_{1} \Sigma^{-1}\right) W_{1}^{*}+G W_{2}^{*} \\
& =L P_{2}^{*} X_{2} \Lambda X_{2}^{\dagger}+G W_{2}^{*},
\end{aligned}
$$

where $G \in C^{k \times(k-s)}$ is an arbitrary matrix.

Substituting $F=L P_{2}^{*}$ and (2.15) into (2.10), we get

$$
X_{1}^{*}\left(L P_{2}^{*} X_{2} \Lambda X_{2}^{\dagger}+G W_{2}^{*}\right)=\left(X_{1} \Lambda\right)^{*} L P_{2}^{*}
$$

By the singular value decomposition of $X_{1}$, then the relation (2.16) becomes

$$
\begin{gathered}
0=S_{2}^{*}\left(X_{1} \Lambda\right)^{*} L P_{2}^{*} \\
D R_{1}^{*}\left(L P_{2}^{*} X_{2} \Lambda X_{2}^{\dagger}+G W_{2}^{*}\right)=S_{1}^{*}\left(X_{1} \Lambda\right)^{*} L P_{2}^{*}
\end{gathered}
$$

Clearly, (2.17) with respect to unknown matrix $L$ is always solvable. From Lemma 2.2 and (2.5), we have

$$
\begin{aligned}
L & =J_{1}-\left(X_{1} \Lambda S_{2}\right)\left(X_{1} \Lambda S_{2}\right)^{\dagger} J_{1} P_{2}^{*} P_{2} \\
& =J_{1}-\left(X_{1} \Lambda S_{2}\right)\left(X_{1} \Lambda S_{2}\right)^{\dagger} J_{1} \\
& =T_{2} J
\end{aligned}
$$

where $J \in C^{(k-g) \times(k-t)}$ is arbitrary. Substituting $L=T_{2} J$ into (2.18), we get

$$
D R_{1}^{*} G W_{2}^{*}=\left(X_{1} \Lambda S_{1}\right)^{*} T_{2} J P_{2}^{*}-D R_{1}^{*} T_{2} J P_{2}^{*} X_{2} \Lambda X_{2}^{\dagger}
$$

Then, we have

$$
R_{1}^{*} G W_{2}^{*}=D^{-1}\left(X_{1} \Lambda S_{1}\right)^{*} T_{2} J P_{2}^{*}-R_{1}^{*} T_{2} J P_{2}^{*} X_{2} \Lambda X_{2}^{\dagger}
$$


Since $R_{1}^{*}$ is of full row rank, then the above equation with respect to $G W_{2}^{*}$ is always solvable. By Lemma 2.2, we have

$$
G W_{2}^{*}=\left(X_{1} \Lambda X_{1}^{\dagger}\right)^{*} T_{2} J P_{2}^{*}-R_{1} R_{1}^{*} T_{2} J P_{2}^{*} X_{2} \Lambda X_{2}^{\dagger}+\left(I-R_{1} R_{1}^{*}\right) Y_{1}
$$

where $Y_{1} \in C^{k \times k}$ is arbitrary. Then, we get

$$
\begin{aligned}
G & =\left(X_{1} \Lambda X_{1}^{\dagger}\right)^{*} T_{2} J P_{2}^{*} W_{2}-R_{1} R_{1}^{*} T_{2} J P_{2}^{*} X_{2} \Lambda X_{2}^{\dagger} W_{2}+\left(I-R_{1} R_{1}^{*}\right) Y_{1} W_{2} \\
& =\left(X_{1} \Lambda X_{1}^{\dagger}\right)^{*} T_{2} J P_{2}^{*} W_{2}+R_{2} Y
\end{aligned}
$$

where $Y \in C^{(k-l) \times(k-s)}$ is arbitrary.

Finally, we have

$$
F=T_{2} J P_{2}^{*}, \quad N=F X_{2} \Lambda X_{2}^{\dagger}+G W_{2}^{*}
$$

where $G=\left(X_{1} \Lambda X_{1}^{\dagger}\right)^{*} F W_{2}+R_{2} Y$. The proof is completed.

From Lemma 2.1, we have that if the mass matrix $M \in H H C^{n \times n}$, then $M$ is not positive definite. If $M$ is symmetric positive definite and $K$ is a symmetric matrix, then (1.3) can be reformulated as the following form:

$$
A X=X \Lambda,
$$

where $A=M^{-1} K$. From [20, Theorem 7.6.3], we know that $A$ is a diagonalizable matrix, all of whose eigenvalues are real. Thus, $\Lambda \in R^{m \times m}$ and $X$ is of full column rank. Assume that $X$ is a real $n \times m$ matrix. Let the singular value decomposition of $X$ be

$$
X=\tilde{U}\left(\begin{array}{l}
\Gamma \\
0
\end{array}\right) \tilde{V}^{T}, \quad \tilde{U} \in O R^{n \times n}, \tilde{V} \in O R^{m \times m}, \Gamma=\operatorname{diag}\left(\gamma_{1}, \ldots, \gamma_{m}\right)>0,
$$

where $O R^{n \times n}$ denotes the set of all orthogonal matrices. The solution of (2.25) can be expressed as

$$
A=\tilde{U}\left(\begin{array}{cc}
\Gamma \tilde{V}^{T} \Lambda \tilde{V} \Gamma^{-1} & Z_{12} \\
0 & Z_{22}
\end{array}\right) \tilde{U}^{T},
$$

where $Z_{12} \in R^{m \times(n-m)}$ is an arbitrary matrix and $Z_{22} \in R^{(n-m) \times(n-m)}$ is an arbitrary diagonalizable matrix (see [21, Theorem 3.1]).

Let $\Lambda=\operatorname{diag}\left(\lambda_{1} I^{k_{1}}, \ldots, \lambda_{q} I^{k_{q}}\right)$ with $\lambda_{1}<\lambda_{2}<\cdots<\lambda_{q}$. Choose $Z_{22}=\bar{G} \Lambda_{2} \bar{G}^{-1}$, where $\bar{G} \in R^{(n-m) \times(n-m)}$ is an arbitrary nonsingular matrix and $\Lambda_{2}=\operatorname{diag}\left(\lambda_{q+1} I^{k_{q+1}}, \ldots, \lambda_{p} I^{k_{p}}\right)$ with $\lambda_{p}>\cdots>\lambda_{q+1}>\lambda_{q}$. The solutions to (1.3) with respect to unknown matrices $M>0$ and $K=K^{T}$ are presented in the following theorem. 
Theorem 2.4 (see [21]). Given that $X \in R^{n \times m}, \operatorname{rank}(X)=m$, and $\Lambda=\operatorname{diag}\left(\lambda_{1} I^{k_{1}}, \ldots, \lambda_{q} I^{k_{q}}\right) \in$ $R^{m \times m}$, let the singular value decomposition of $X$ be (2.26). Then the symmetric positive-definite solution $M$ and symmetric solution $K$ to (1.3) can be expressed as

$$
M=\tilde{U} \tilde{F}^{T} \tilde{F} \tilde{U}^{T}, \quad K=\tilde{U} \tilde{F}^{T} \Delta \tilde{F} \tilde{U}^{T}
$$

where $\Delta=\operatorname{diag}\left(\Lambda, \Lambda_{2}\right), \tilde{F}=\left(\begin{array}{cc}F_{11} & F_{12} \\ 0 & F_{22}\end{array}\right), F_{11}=\operatorname{diag}\left(L_{1}, \ldots, L_{q}\right) V \Gamma^{-1} \in R^{m \times m}$, and $F_{22}=$ $\operatorname{diag}\left(L_{q+1}, \ldots, L_{p}\right) \bar{G}^{-1} \in R^{(n-m) \times(n-m)}$, where $L_{i} \in R^{k_{i} \times k_{i}}$ is an arbitrary nonsingular matrix $(i=1,2, \ldots, p)$. The matrix $F_{12}$ satisfies the equation $\Lambda F_{12} \bar{G}-F_{12} \bar{G} \Lambda_{2}=F_{11} Z_{12} \bar{G}$.

\section{Solution of Problem 2}

Lemma 3.1 (see [22]). Given that $A \in C^{m \times n}, B \in C^{p \times q}, C \in C^{l \times n}, D \in C^{p \times t}, E \in C^{m \times q}$, and $H \in C^{l \times t}$, let

$$
\begin{gathered}
S_{a}=\left\{Z \mid Z \in C^{n \times p},\|[A Z B-E, C Z D-H]\|^{2}=\min \right\}, \\
S_{b}=\left\{Z \mid Z \in C^{n \times p}, A^{*} A Z B B^{*}+C^{*} C Z D D^{*}=A^{*} E B^{*}+C^{*} H D^{*}\right\} .
\end{gathered}
$$

Then $Z \in S_{a}$ if and only if $Z \in S_{b}$.

For the given matrices $K_{a}, M_{a} \in H C^{n \times n}$, let

$$
U^{*} M_{a} U=\left(\begin{array}{ll}
C_{1} & C_{2} \\
C_{2}^{*} & C_{3}
\end{array}\right), \quad U^{*} K_{a} U=\left(\begin{array}{ll}
K_{1} & K_{2} \\
K_{2}^{*} & K_{3}
\end{array}\right)
$$

From Theorem 2.3, we know that $S_{E} \neq \emptyset$. The following theorem is for the best approximation solution of Problem 2.

Theorem 3.2. Given that $X \in C^{n \times m}, \Lambda \in C^{m \times m}$, and $K_{a}, M_{a} \in H C^{n \times n}$, then Problem 2 has a unique solution and the solution can be expressed as

$$
\widehat{M}=U\left(\begin{array}{cc}
0 & \widehat{F} \\
\widehat{F}^{*} & 0
\end{array}\right) U^{*}, \quad \widehat{K}=U\left(\begin{array}{cc}
0 & \hat{F} X_{2} \Lambda X_{2}^{\dagger}+K_{2} W_{2} W_{2}^{*} \\
\left(\widehat{F} X_{2} \Lambda X_{2}^{\dagger}+K_{2} W_{2} W_{2}^{*}\right)^{*} & 0
\end{array}\right) U^{*},
$$

where

$$
\widehat{F}=\left(C_{2}+K_{2}\left(X_{2} \Lambda X_{2}^{\dagger}\right)^{*}\right)\left(I+\left(X_{2} \Lambda X_{2}^{\dagger}\right)\left(X_{2} \Lambda X_{2}^{\dagger}\right)^{*}\right)^{-1}
$$


Proof. It is easy to verify that $S_{E}$ is a closed convex subset of $H H C^{n \times n} \times H H C^{n \times n}$. From the best approximation theorem, we know that there exists a unique solution $(\widehat{K}, \widehat{M})$ in $S_{E}$ such that (1.4) holds. From Theorem 2.3 and the unitary invariant of the Frobenius norm, we have

$$
\begin{aligned}
& \left\|M_{a}-M\right\|^{2}+\left\|K_{a}-K\right\|^{2} \\
& \quad=\left\|\left(\begin{array}{ll}
C_{1} & C_{2} \\
C_{2}^{*} & C_{3}
\end{array}\right)-\left(\begin{array}{cc}
0 & F \\
F^{*} & 0
\end{array}\right)\right\|^{2}+\left\|\left(\begin{array}{cc}
K_{1} & K_{2} \\
K_{2}^{*} & K_{3}
\end{array}\right)-\left(\begin{array}{cc}
0 & F X_{2} \Lambda X_{2}^{\dagger}+G W_{2}^{*} \\
\left(F X_{2} \Lambda X_{2}^{\dagger}+G W_{2}^{*}\right)^{*} & 0
\end{array}\right)\right\|^{2},
\end{aligned}
$$

where $G=\left(X_{1} \Lambda X_{1}^{\dagger}\right)^{*} F W_{2}+R_{2} Y$. Hence, $\left\|M_{a}-M\right\|^{2}+\left\|K_{a}-K\right\|^{2}=$ min is equivalent to

$$
\left\|F-C_{2}\right\|^{2}+\left\|F X_{2} \Lambda X_{2}^{\dagger}+\left(X_{1} \Lambda X_{1}^{\dagger}\right)^{*} F W_{2} W_{2}^{*}+R_{2} Y W_{2}^{*}-K_{2}\right\|^{2}=\min .
$$

Let

$$
f=\left\|F-C_{2}\right\|^{2}+\left\|F X_{2} \Lambda X_{2}^{\dagger}+\left(X_{1} \Lambda X_{1}^{\dagger}\right)^{*} F W_{2} W_{2}^{*}+R_{2} Y W_{2}^{*}-K_{2}\right\|^{2}
$$

Then from the unitary invariant of the Frobenius norm, we have

$$
\begin{aligned}
f= & \left\|F-C_{2}\right\|^{2} \\
& +\left\|F X_{2} \Lambda X_{2}^{\dagger}\left(W_{1}, W_{2}\right)+\left(X_{1} \Lambda X_{1}^{\dagger}\right)^{*} F W_{2} W_{2}^{*}\left(W_{1}, W_{2}\right)+R_{2} Y W_{2}^{*}\left(W_{1}, W_{2}\right)-K_{2}\left(W_{1}, W_{2}\right)\right\|^{2} \\
= & \left\|F-C_{2}\right\|^{2}+\left\|\left(F X_{2} \Lambda X_{2}^{\dagger} W_{1}, 0\right)+\left(0,\left(X_{1} \Lambda X_{1}^{\dagger}\right)^{*} F W_{2}\right)+\left(0, R_{2} Y\right)-\left(K_{2} W_{1}, K_{2} W_{2}\right)\right\|^{2} \\
= & \left\|F-C_{2}\right\|^{2}+\left\|F X_{2} \Lambda X_{2}^{\dagger} W_{1}-K_{2} W_{1}\right\|^{2}+\left\|\left(X_{1} \Lambda X_{1}^{\dagger}\right)^{*} F W_{2}+R_{2} Y-K_{2} W_{2}\right\|^{2} .
\end{aligned}
$$

Let $h=\left\|\left(X_{1} \Lambda X_{1}^{\dagger}\right)^{*} F W_{2}+R_{2} Y-K_{2} W_{2}\right\|^{2}$. It is not difficult to see that, when

$$
R_{2} Y=K_{2} W_{2}-\left(X_{1} \Lambda X_{1}^{\dagger}\right)^{*} F W_{2}
$$


that is, $Y=R_{2}^{*} K_{2} W_{2}-R_{2}^{*}\left(X_{1} \Lambda X_{1}^{\dagger}\right)^{*} F W_{2}$, we have $h=0$. In other words, we can always find $Y$ such that $h=0$. Let

$$
g=\left\|F-C_{2}\right\|^{2}+\left\|F X_{2} \Lambda X_{2}^{\dagger} W_{1}-K_{2} W_{1}\right\|^{2}=\left\|\left[F-C_{2}, F X_{2} \Lambda X_{2}^{\dagger} W_{1}-K_{2} W_{1}\right]\right\|^{2} .
$$

Then, we have that $f=\min$ is equivalent to $g=\min$. According to Lemma 3.1 and (3.10), we get the following matrix equation:

$$
F+F\left(X_{2} \Lambda X_{2}^{\dagger} W_{1}\right)\left(X_{2} \Lambda X_{2}^{\dagger} W_{1}\right)^{*}=C_{2}+K_{2} W_{1}\left(X_{2} \Lambda X_{2}^{\dagger} W_{1}\right)^{*}
$$

and its solution is $\widehat{F}=\left(C_{2}+K_{2}\left(X_{2} \Lambda X_{2}^{\dagger}\right)^{*}\right)\left(I+\left(X_{2} \Lambda X_{2}^{\dagger}\right)\left(X_{2} \Lambda X_{2}^{\dagger}\right)^{*}\right)^{-1}$. Again from Lemma 3.1, we have that, when $F=\widehat{F}, g$ attains its minimum, which gives $\hat{Y}=R_{2}^{*} K_{2} W_{2}-$ $R_{2}^{*}\left(X_{1} \Lambda X_{1}^{\dagger}\right)^{*} \widehat{F} W_{2}$, and $\widehat{G}=\left(X_{1} \Lambda X_{1}^{\dagger}\right)^{*} \widehat{F} W_{2}+R_{2} \widehat{Y}=K_{2} W_{2}$. Then, the unique solution of Problem 2 given by (3.3) is obtained.

2.

Now, we give an algorithm to compute the optimal approximate solution of Problem

\section{Algorithm.}

(1) Input $K_{a}, M_{a}, X, \Lambda$, and $U$.

(2) Compute $X_{2}$ according to (2.3).

(3) Find the singular value decomposition of $X_{2}$ according to (2.4).

(4) Calculate $\widehat{F}$ by (3.4).

(5) Compute $(\widehat{M}, \widehat{K})$ by (3.3).

Example 1. Let $n=6, m=3$, and the matrices $M_{a}, K_{a}, X$, and $\Lambda$ be given by

$$
\begin{aligned}
M_{a} & =\left(\begin{array}{cccccc}
1.56 & 0.66 & 0.54 & -0.39 & 0 & 0 \\
0.66 & 0.36 & 0.39 & -0.27 & 0 & 0 \\
0.54 & 0.39 & 3.12 & 0 & 0.54 & -0.39 \\
-0.39 & -0.27 & 0 & 0.72 & 0.39 & -0.27 \\
0 & 0 & 0.54 & 0.39 & 3.12 & 0 \\
0 & 0 & -0.39 & -0.27 & 0 & 0.72
\end{array}\right), \\
K_{a} & =\left(\begin{array}{cccccc}
2 & 3 & -2 & 3 & 0 & 0 \\
3 & 6 & -3 & 3 & 0 & 0 \\
-2 & -3 & 4 & 0 & -2 & 3 \\
3 & 3 & 0 & 12 & -3 & 3 \\
0 & 0 & -2 & -3 & 4 & 0 \\
0 & 0 & 3 & 3 & 0 & 12
\end{array}\right),
\end{aligned}
$$




$$
\begin{aligned}
X & =\left(\begin{array}{ccc}
0.0347+0.1507 i & -0.6975 i & 0.0003+0.0858 i \\
0.6715 i & 0.0277+0.0760 i & -0.0846-0.0101 i \\
-0.0009+0.1587 i & -0.0814+0.0196 i & 0.6967 \\
-0.1507+0.0347 i & 0.6975 & -0.0858+0.0003 i \\
-0.6715 & -0.0760+0.0277 i & 0.0101-0.0846 i \\
-0.1587-0.0009 i & -0.0196-0.0814 i & 0.6967 i
\end{array}\right), \\
\Lambda & =\operatorname{diag}(0.3848+0.0126 i, 2.5545+0.4802 i, 2.5607) .
\end{aligned}
$$

From the Algorithm, we obtain the unique solution of Problem 2 as follows:

$$
\begin{gathered}
\widehat{F}=\left(\begin{array}{ccc}
-1.4080+1.1828 i & 1.0322+0.4732 i & -0.8111-0.0874 i \\
0.9537+0.2935 i & -0.7529-0.0137 i & -0.6596-0.3106 i \\
-0.6624+0.1982 i & -0.3566-0.0051 i & -1.0958+1.0040 i
\end{array}\right), \\
\widehat{N}=\left(\begin{array}{ccc}
-4.3706+2.1344 i & 1.6264-0.3128 i & -2.2882-0.3290 i \\
2.4251+1.2137 i & -0.5229+0.0005 i & -1.4620-0.7688 i \\
-1.6669+0.1663 i & 0.6991-0.6057 i & -2.6437+2.5190 i
\end{array}\right), \\
\widehat{M}=U\left(\begin{array}{cc}
0 & \widehat{F} \\
\widehat{F}^{*} & 0
\end{array}\right) U^{*}, \quad \widehat{K}=U\left(\begin{array}{cc}
0 & \widehat{N} \\
\widehat{N} & 0
\end{array}\right) U^{*},
\end{gathered}
$$

where $U=(1 / \sqrt{2})\left(\begin{array}{cc}I_{3} & I_{3} \\ -i I_{3} & i I_{3}\end{array}\right)$. It is easy to calculate $\|\widehat{K} X-\widehat{M} X \Lambda\|=2.1121 e-015$, and $\|[\widehat{M}-$ $\left.M_{a}, \widehat{K}-K_{a}\right] \|=19.7467$.

\section{Acknowledgments}

This paper was granted financial support from National Natural Science Foundation (10901056) and Shanghai Natural Science Foundation (09ZR1408700), NSFC grant (10971070). The authors would like to thank the referees for their valuable comments and suggestions.

\section{References}

[1] M. I. Friswell and J. E. Mottershead, Finite Element Model Updating in Structural Dynamics, vol. 38 of Solid Mechanics and Its Applications, Kluwer Academic Publishers, Dordrecht, The Netherlands, 1995.

[2] A. Berman, "Mass matrix correction using an incomplete set of measured modes," AIAA Journal, vol. 17, pp. 1147-1148, 1979.

[3] F.-S. Wei, "Stiffness matrix correction from incomplete test data," AIAA Journal, vol. 18, pp. 1274-1275, 1980.

[4] M. I. Friswell, D. J. Inman, and D. F. Pilkey, "Direct updating of damping and stiffness matrices," AIAA Journal, vol. 36, no. 3, pp. 491-493, 1998.

[5] Y.-C. Kuo, W.-W. Lin, and S.-F. Xu, "New methods for finite element model updating problems," AIAA Journal, vol. 44, no. 6, pp. 1310-1316, 2006. 
[6] Y.-C. Kuo, W.-W. Lin, and S.-F. Xu, "New model correcting method for quadratic eigenvalue problems using symmetric eigenstructure assignment," AIAA Journal, vol. 43, no. 12, pp. 2593-2598, 2005.

[7] W. Luber and A. Lotze, "Application of sensitivity methods for error localization in finite element systems," in Proceedings of the 8th International Modal Anlysis Conference, pp. 598-604, 1990.

[8] M. Link, "Identification and correction of errors in analytical models using test data-theoretical and practical bounds," in Proceedings of the 8th International Modal Anlysis Conference, pp. 570-578, 1990.

[9] J.-C. O'Callahan and C.-M. Chou, "Localization of model errors in optimized mass and stiffness matrices using model test data," International Journal of Analytical and Experimental Analysis, no. 4, pp. 8-14, 1989.

[10] R. G. Cobb and B. S. Liebst, "Structural damage identification using assigned partial eigenstructure," AIAA Journal, vol. 35, no. 1, pp. 152-158, 1997.

[11] Y.-X. Yuan, "A symmetric inverse eigenvalue problem in structural dynamic model updating," Applied Mathematics and Computation, vol. 213, no. 2, pp. 516-521, 2009.

[12] Y.-X. Yuan and H. Dai, "A generalized inverse eigenvalue problem in structural dynamic model updating," Journal of Computational and Applied Mathematics, vol. 226, no. 1, pp. 42-49, 2009.

[13] O.-C. Zienkiewicz, The Finite Element Method, McGraw-Hill, London, UK, 3rd edition, 1977.

[14] D. Xie, X. Hu, and L. Zhang, "The solvability conditions for inverse eigenproblem of symmetric and anti-persymmetric matrices and its approximation," Numerical Linear Algebra with Applications, vol. 10, no. 3, pp. 223-234, 2003.

[15] D. Xie and Y. Sheng, "Inverse eigenproblem of anti-symmetric and persymmetric matrices and its approximation," Inverse Problems, vol. 19, no. 1, pp. 217-225, 2003.

[16] Z.-J. Bai and R. H. Chan, "Inverse eigenproblem for centrosymmetric and centroskew matrices and their approximation," Theoretical Computer Science, vol. 315, no. 2-3, pp. 309-318, 2004.

[17] W. F. Trench, "Inverse eigenproblems and associated approximation problems for matrices with generalized symmetry or skew symmetry," Linear Algebra and Its Applications, vol. 380, no. 1-3, pp. 199-211, 2004.

[18] Y.-X. Yuan, "Inverse eigenproblem for $R$-symmetric matrices and their approximation," Journal of Computational and Applied Mathematics, vol. 233, no. 2, pp. 308-314, 2009.

[19] A. Ben-Israsel and T. N. E. Grecille, Generalized Inverse Theory and Application, Springer, Berlin, Germany, 2nd edition, 2003.

[20] R. A. Horn and C. R. Johnson, Matrix Analysis, Cambridge University Press, Cambridge, UK, 1985.

[21] J. G. Sun, "Two kinds of inverse eigenvalue problems for real symmetric matrices," Mathematica Numerica Sinica, vol. 10, no. 3, pp. 282-290, 1988 (Chinese)

[22] Y.-X. Yuan, "Least squares solutions of matrix equation $A X B=E, C X D=F$," Journal of East China Shipbuilding Institute, vol. 18, no. 3, pp. 29-31, 2004 (Chinese). 


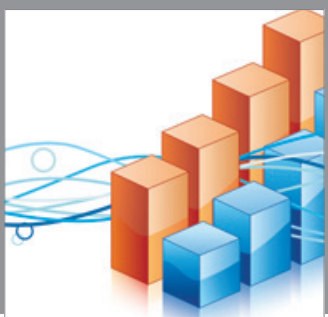

Advances in

Operations Research

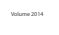

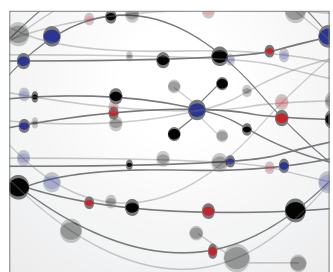

\section{The Scientific} World Journal
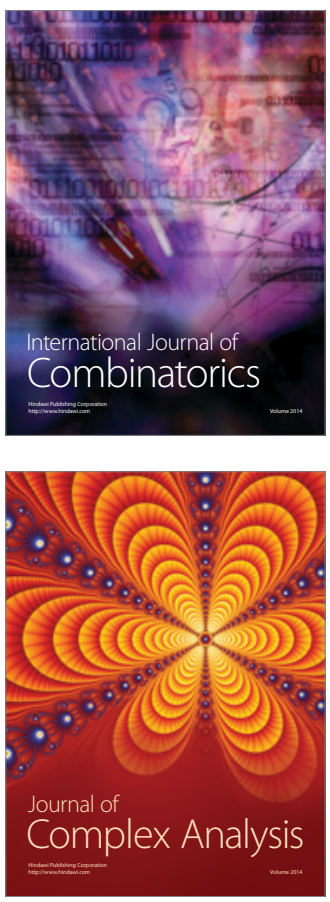

International Journal of

Mathematics and

Mathematical

Sciences
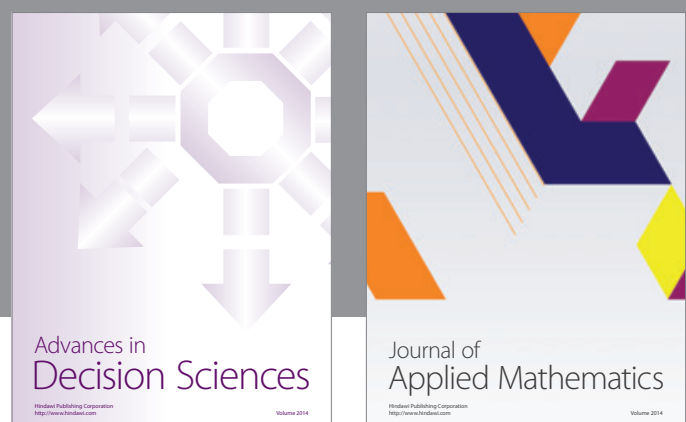

Journal of

Applied Mathematics
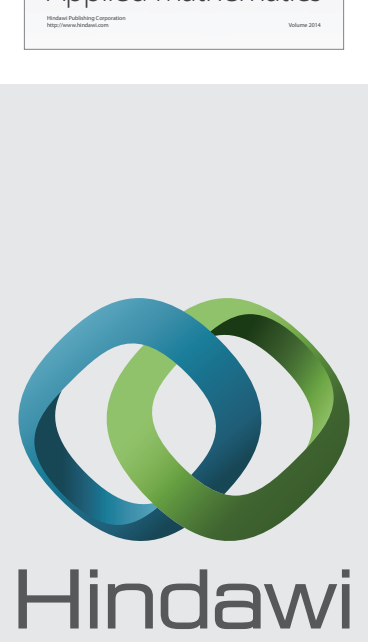

Submit your manuscripts at http://www.hindawi.com
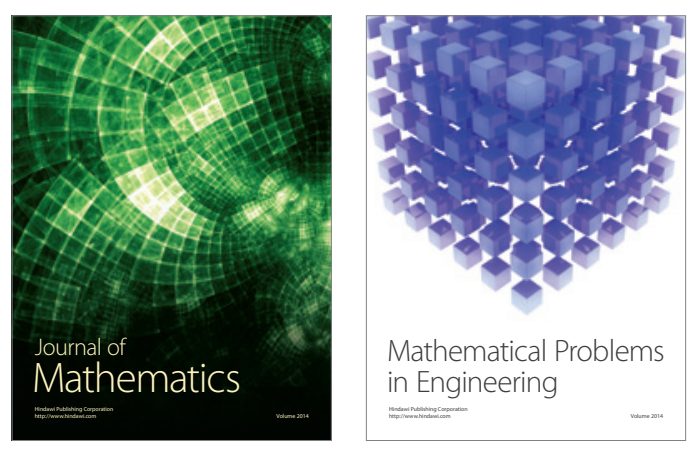

Mathematical Problems in Engineering
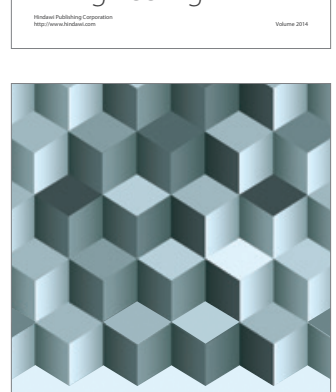

Journal of

Function Spaces
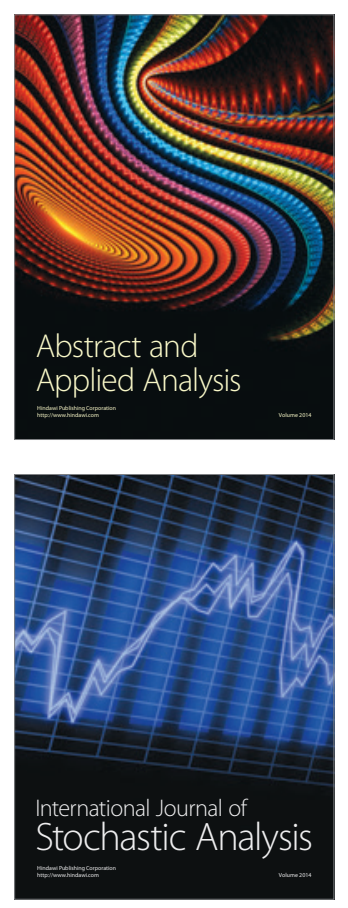

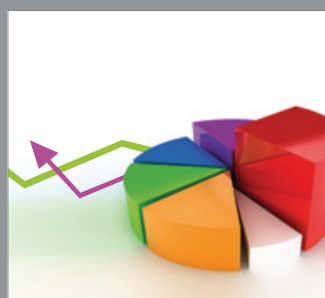

ournal of

Probability and Statistics

Promensencen
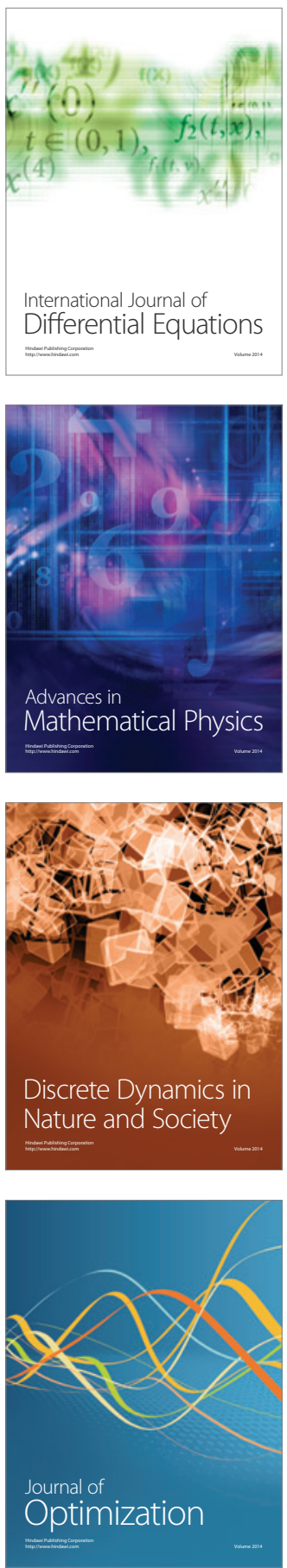